\title{
AUTOMATIC CONTINUITY OF POSITIVE FUNCTIONALS ON TOPOLOGICAL INVOLUTION ALGEBRAS
}

\author{
P.G, DIXON
}

This paper surveys the known results on automatic continuity of positive functionals on topological *algebras and then shows how two theorems on Banach *-algebras extend to complete metrizable topological *algebras. The two theorems concerned are Loy's theorem on separable Banach *-algebras $A$ with centre 2 such that $A Z$ is of countable codimension and Varopoulos' result on Banach *-algebras with bounded approximate identity. Both theorems have the conclusion that all positive functionals on such algebras are continuous. The extension of the second theorem requires the algebra to be locally convex and the approximate identity to be 'uniformly bounded'. Neither extension requires the algebra to be LMC. This means that the proof of the first theorem is quite different from the corresponding Banach algebra result (which used spectral theory). The proof of the second is closer to the previously known LMC version, but actually neater by being more general. It is also shown that the well-known estimate of $\left|f\left(a^{*} b a\right)\right|$ for a positive functional $f$ on a Banach *-algebra may be obtained without the usual use of spectral theory. The paper concludes with a list of open questions.

\section{Introduction}

The purpose of this paper is to give analogues for complete metrizable algebras of the following two theorems on Banach algebras.

Received 22 October 1980. 
THEOREM 1.1 (Loy [12, Theorem 2.1]). Let $A$ be a separable Banach algebra with involution and let $Z$ be the centre of $A$. If $A Z$ is of countable (that is, at most countable) codimension in $A$, then every positive functional on $A$ is continuous.

THEOREM 1.2 (Varopoulos [21]). Let $A$ be a Banach algebra with involution. If $A$ has a bounded approximate identity, then every positive functional on $A$ is continuous.

Theorem 1.1 is the end product of a series of results. It is based on an application of Souslin techniques to earlier results on (not necessarily separable) algebras (Theorem 1.4 below). Theorem 1.2 may be found in Sinclair's book [19, Theorem 13.11]. Both these theorems hold for Banach algebras whose involutions are not necessarily continuous, whereas our proofs will require continuous involutions. However, it should be noted that involutions on semisimple Banach algebras are automatically continuous [19, Corollary 6.13], so this may be regarded as only a minor loss. Apart from this, and an adjustment to the notion of 'bounded approximate identity', our results (Theorems 3.6 and 4.3 below) are verbatim extensions of these theorems. Our proofs, however, are substantially different.

Let us establish some terminology. All topological vector spaces will be Hausdorff. All algebras will be over the complex field C . Involutions will not be assumed continuous unless specifically stated. By an $F$-space we shall mean a complete metrizable (not necessarily locally convex) topological vector space. By an $F$-algebra we shall mean a topological algebra which is an $F$-space. The multiplication is assumed to be separately continuous, but a theorem of Arens [1] ([22, Theorem 7.2]) then makes it jointly continuous; that is, the map $(x, y) \mapsto x y$ from $A \times A$ to $A$ is continuous. If $X, Y$ are subspaces of $A$, then $X Y$ denotes the subspace generated by the appropriate set of products; that is,

$$
X Y=\left\{\sum_{i=1}^{n} x_{i} y_{i}: n=1,2,3, \ldots ; x_{i} \in X, y_{i} \in Y(1 \leq i \leq n)\right\} .
$$

We write $A^{2}$ for $A A$. By an $F^{*}$-algebra we shall mean an $F$-algebra with a continuous involution. The positive cone of a ${ }^{*}$-algebra $A$ is the set 


$$
A^{+}=\left\{\sum_{i=1}^{n} x_{i}^{*} x_{i}: n=1,2,3, \ldots ; x_{i} \in A(1 \leq i \leq n)\right\} \text {. }
$$

A positive functional on $A$ is a linear map $f: A \rightarrow \mathbb{C}$ such that $f\left(x^{*} x\right) \geq 0$ for all $x \in A$; that is, such that $f\left(A^{+}\right) \subseteq \mathbb{R}^{+}$.

With these notations, we summarize the other previously known results about automatic continuity of positive functionals on topological *algebras. First, a trivial argument with Hamel bases shows the following.

THEOREM 1.3. A necessary condition for an F-algebra $A$ with involution to have no discontinuous positive functionals is that $A^{2}$ be closed and of finite codimension in $A$.

In the other direction, we have the following.

THEOREM 1.4 ([19, Theorem 13.7]). Let $A$ be a Banach algebra with involution, and let $z$ be the centre of $A$. If $Z^{2} A$ is closed and of finite codimension in $A$, then every positive functional on $A$ is continuous.

THEOREM 1.5. Let $A$ be a complete metrizable algebra with involution. If $A^{2}$ is closed and of finite codimension and $A^{+}$is elosed, then every positive functional on $A$ is continuous.

This is an easy corollary of a result of Namioka [15, Corollary 5.5]. In the following extension of $i t$, due to Neumann $[16,(3.6)(i i)]$, the involution need not even satisfy the identity $(x y)^{*}=y^{*} x^{*}(x, y \in A)$.

THEOREM 1.6. Let $A$ be a complete metrizable algebra with involution such that $A^{2}$ is of countable codimension in $A$ and $A^{+}$is complete. (It is sufficient, in fact, to assume that every increasing Cauchy sequence in $A^{+}$converges there.) Then $A^{2}$ is closed and of finite codimension in $A$ and every positive functional on $A$ is continuous.

THEOREM 1.7 (Dao-Shing Shah [18]). If $A$ is an $F^{*}$-algebra with identity, then every positive functional on $A$ is continuous.

Actually, Shah proves this only for LMC algebras, but his method 
extends easily to the general case (see [7, Theorem (I.8.5)], [6, Theorem 11.1] or [17]).

If we drop the hypothesis of metrizability, even Shah's theorem fails easily (see [14, remark following Proposition 12.2]), since the question we should be asking is whether positive functionals are automatically bounded. This automatic boundedness can be proved for complete LMC algebras with continuous involution and identity ([7, Theorem (I.8.6)], [9]), but it fails in general. In [8, Addendum], an example is given of a complete, commutative, locally convex algebra with identity, continuous involution and jointly continuous multiplication, on which there is an unbounded positive functional.

Finally, we mention two very recent results of Johnson [11, Theorems 3.5 and 3.7]. For $F^{*}$-algebras, these read as follows.

THEOREM 1.8. Let $A$ be an $F^{*}$-algebra such that $A^{2}=A$. Suppose either:

(a) A is commutative and LMC and the set of elements with bounded spectrum is dense in $A$; or

(b) there is an approximate identity $\left\{e_{\lambda}\right\}_{\lambda \in \Lambda}$ for $A$, which is contained in the centre of $A$ and is such that the set $\left\{e_{\lambda} x: \lambda \in \Lambda\right\}$ is bounded, for every $x \in A$.

Then every positive functional on $A$ is continuous.

\section{Results in general $F^{*}$-algebras}

In this section, we present the best result on $F^{*}$-algebras we have been able to reach, so far, without invoking the hypothesis of separability (Theorem 2.5). We begin with a result which is basic to the rest of the paper. For Banach algebras with involution, it may be proved using Ford's square root lemma (see [19, Lemma 13.2 (iv)]). In general topological algebras Ford's lemma is not available, so we must use Shah's theorem, which avoids the need to consider spectra of elements, at the expense of requiring us to assume that the involution is continuous; hence the prevalence of this assumption in the present paper.

THEOREM 2.1. Let $A$ be an $F^{*}$-algebra and $f$ a positive functional 
on $A$. Then, for every $a, b \in A$, the functional $x \mapsto f(a x b)$ on $A$ is continuous.

Proof. Using the identity

$4 a x b=\left(b+a^{*}\right) * x\left(b+a^{*}\right)+i\left(b+i a^{*}\right) * x\left(b+i a^{*}\right)$

$$
-\left(b-a^{*}\right)^{*} x\left(b-a^{*}\right)-i\left(b-i a^{*}\right)^{*} x\left(b-i a^{*}\right),
$$

we see that it is sufficient to prove that the functional

$f_{a}: x \mapsto f\left(a^{\star} x a\right)$ is continuous, for each $a \in A$.

Let $A^{\sim}$ be the algebra $A \oplus \mathbb{C} e$ obtained by adjoining an identity $e$ to $A$ in the usual way, with the obvious topology. Then $f_{a}$ extends to a positive functional on $A^{\sim}$ by

$$
f_{a}(x+\lambda e)=f\left(a^{*} x a\right)+\lambda f\left(a^{*} a\right) \quad(x \in A, \lambda \in \mathbb{C}) .
$$

By Shah's theorem (Theorem 1.7 above), $f_{a}$ is continuous on $A^{\sim}$, and hence on $A$.

An interesting corollary to this is that we may obtain the usual estimate for the norm of $f$ when $A$ is a Banach algebra [19, Lemma 13.2 (iv)], without using Ford's square root lemma. En route to this, we first obtain a result for general $F^{*}$-algebras.

THEOREM 2.2. Let $f$ be a positive functional on an $F^{*}$-algebra $A$. If.$x \in A$ is such that $\left(x x^{*}\right)^{n} \rightarrow 0$ (equivalently $\left(x^{*} x\right)^{n} \rightarrow 0$ ), as $n \rightarrow \infty$, then

$$
\left|f\left(a^{*} x a\right)\right| \leq f\left(a^{*} a\right) \quad(a \in A) .
$$

Proof. We need the following algebraic lemma.

LEMMA 2.3. If $f$ is a positive functional on a *-algebra $A$, then

$$
|f(x y z)|^{2^{n+1}} \leq f\left(x x^{*}\right)^{2^{n}-1} f\left(x\left(y y^{*}\right)^{2^{n}} x^{*}\right) f\left(z^{*} z\right)^{2^{n}}
$$

for $n=0,1,2, \ldots$ and every $x, y, z \in A$.

Proof. We start with the fact that $f$ must satisfy the CauchySchwarz inequality

$$
\left|f\left(a^{*} b\right)\right|^{2} \leq f\left(a^{*} a\right) f\left(b^{*} b\right) \quad(a, b \in A) .
$$

Putting $a^{*}=x y, b=z$ in this gives 


$$
|f(x y z)|^{2} \leq f\left(x y y^{*} x^{*}\right) f\left(z^{*} z\right),
$$

which proves the lemma for $n=0$. Furthermore, (1) implies

$$
f\left(x\left(y y^{*}\right)^{2^{n}} x^{*}\right)^{2} \leq f\left(x\left(y y^{*}\right)^{2^{n+1}} x^{*}\right) f\left(x x^{*}\right)
$$

for all $n$. Now suppose the lemma holds for $n$. Then

$$
\begin{aligned}
|f(x y z)|^{2^{n+2}} & \leq\left[f\left(x x^{*}\right)^{2^{n}-1} f\left(x\left(y y^{*}\right)^{2^{n}} x^{*}\right) f\left(z^{*} z\right)^{2^{n}}\right]^{2} \\
& \leq f\left(x x^{*}\right)^{2^{n+1}-1} f\left(x\left(y y^{*}\right)^{2^{n+1}} x^{*}\right) f\left(z^{*} z\right)^{2^{n+1}},
\end{aligned}
$$

by (2). Thus the lemma is proved, by induction.

Proof of Theorem 2.2 continued. By Theorem 2.1, we have

$$
f\left(a^{*}\left(x x^{*}\right)^{2^{n}} a\right)+0
$$

as $n \rightarrow \infty$ and, by the lemma,

(4) $\quad\left|f\left(a^{*} x a\right)\right|^{2^{n}} \leq f\left(a^{*} a\right)^{2^{n}-1} f\left(a^{*}\left(x x^{*}\right)^{2^{n-1}} a\right) \quad(n=1,2,3, \ldots)$.

If $f\left(a^{*} a\right)=0$, then (4) implies $f\left(a^{*} x a\right)=0$, as desired. Otherwise,

(3) and (4) give

$$
\left|\frac{f\left(a^{*} x a\right)}{f\left(a^{*} a\right)}\right|^{2^{n}} \rightarrow 0
$$

as $n \rightarrow \infty$, so

$$
\left|\frac{f\left(a^{*} x a\right)}{f\left(a^{*} a\right)}\right|<1
$$

This completes the proof.

COROLLARY 2.4 ([19, Lemma 13.2 (iv)]). If $f$ is a positive functional on a Bconach algebra $A$ with continuous involution, then

$$
\left|f\left(a^{*} b a\right)\right| \leq f\left(a^{*} a\right) v\left(b^{*} b\right)^{\frac{1}{2}} \quad(a, b \in A),
$$

where $v(x)$ denotes the spectral radius of $x$. Equivalently [19, Lemma 13.2 (iii)],

$$
\left|f\left(a^{*} h a\right)\right| \leq f\left(a^{*} a\right) \cup(h) \quad\left(a \in A, h=h^{*} \in A\right) \text {. }
$$

Proof. This is immediate from Theorem 2.2, since $\left(b^{*} b\right)^{n} \rightarrow 0$ as 
$n+\infty$ when $v\left(b^{*} b\right)<1$.

Returning to $F^{*}$-algebras, we can develop Theorem 2.1 further, as follows.

THEOREM 2.5. If $f$ is a positive functional on an $F^{*}$-algebra $A$ with centre 2 , then the mapping

$$
(x, y, z) \mapsto f(x y z): A \times A \times z \rightarrow \mathbb{C}
$$

is continuous.

Proof. Since $x y z=x z y=z x y$, Theorem 2.1 implịes that

$$
x \mapsto f(x y z), y \mapsto f(x y z), z \mapsto f(x y z)
$$

are continuous maps from $A$ to $\mathbb{C}$, for all values of the other two variables. It only remains to apply a 'separate continuity implies joint continuity' lemma similar to the theorem of Arens cited above. The following has sufficient generality for our purpose. Its proof is a straightforward generalization of that of Arens' original theorem (see [1] or [22, Theorem 7.2]), and is therefore omitted.

LEMMA 2.6. Let $E_{1}, E_{2}, \ldots, E_{n}$ be $F$-spaces and let $E$ be any topological vector space. Let $T: E_{1} \times \ldots \times E_{n} \rightarrow F$ be a multilinear map, separately continuous in each variable. (That is, each of the maps

$$
x_{i} \mapsto T\left(x_{1}, \ldots, x_{n}\right): E_{i} \rightarrow F \quad\left(x_{j} \in E_{j}(j \neq i), 1 \leq i \leq n\right)
$$

is continuous.) Then $T$ is (jointly) continuous.

\section{Separable $F^{*}$-algebras}

When the $F^{*}$-algebra is separable, we may improve on the results of the previous section by using the theory of analytic spaces. We first recall the basic definitions and results we shall need. For details, we refer the reader to [3], [13], [10] or, with slightly different terminology, [2].

DEFINITION 3.1. A topological space is analytic if it is the continuous image of a complete, separable metric space.

THEOREM 3.2 ([3, Theorem 5.5]). Let $E$ be an F-space and $F$ an conalytic (linear) subspace of countable codimension. Then $F$ is closed 
and of finite codimension.

THEOREM 3.3 (The Pettis Lemma [3, Theorem 5.1]). Let $E$ be a topological vector space and let $X$ be a subset of $E$ which is analytic and non-meagre in $E$. Then $X-X$ (that is, $\{x-y: x, y \in X\}$ ) is a neighbourhood of zero in $E$.

THEOREM 3.4 (The Analytic Graph Theorem [3, Theorem 5.2]). Let $E$ be an E-space, $F$ any topological vector space and $T: E \rightarrow F$ a linear mapping whose graph is an analytic subspace of $E \times F$. Then $T$ is continuous.

We shall express our use of analytic space techniques in the form of a general lemma (which may be of independent interest). Part ( $i$ ) is very similar to the main result of [4] and to [12, Theorem 1.3 (i)], but we include it, for completeness.

LEMMA 3.5. (i) Let $E_{1}, \ldots, E_{n}, E$ be separable F-spaces. Let $T: E_{1} \times \ldots \times E_{n} \rightarrow E$ be a continuous multilinear mapping such that the linear span $H$ of $T\left(E_{1} \times \ldots \times E_{n}\right)$ is of countable codimension in $E$. Then $H$ is closed and of finite codimension and there is a positive integer $K$ such that every element of $H$ is expressibie in the form $\sum_{i=1}^{K} T\left(x_{i 1}, \ldots, x_{i n}\right)$ for some $x_{i j} \in E_{j} \quad(1 \leq i \leq K, 1 \leq j \leq n)$.

(ii) If, further, $F$ is another $F$-space and $S: E \rightarrow E$ is a linear map such that $S T: E_{1} \times \ldots \times E_{n} \rightarrow F$ is (jointly) continuous, then $S$ is continuous.

Proof. Let $H_{k}$ be the set of elements of $H$ expressible in the form $\sum_{i=1}^{k} T\left(x_{i 1}, \ldots, x_{i n}\right)$ for some $x_{i j} \in E_{j}(1 \leq i \leq k, 1 \leq j \leq n)$. Then each $H_{k}$ is analytic, being a continuous image of the complete, separable metric space $\left(E_{1} \times \ldots \times E_{n}\right)^{k}$, and $H$ is analytic, being a continuous image of the complete, separable metric space consisting of the disjoint union $\bigcup_{k=1}^{\infty}\left(E_{1} \times \ldots \times E_{n}\right)^{k}$. By Theorem 3.2, $H$ is closed and of finite 
codimension in $E$. So $H$ is a complete metric space. Moreover, $H=\bigcup_{k=1}^{\infty} H_{k}$ so, by Baire's Category Theorem, $H_{k}$ is non-meagre in $H$, for some $k$. Applying the Pettis Lemma to this $H_{k}$, we see that $H_{k}-H_{k}$ is a zero-neighbourhood in $H$. Since $H_{k}$ is a linear subspace, this means that $H_{k}-H_{k}=H$; that is, $H_{2 k}=H$. This proves (i) with $K=2 k$.

To prove (ii), we first consider the restriction $S \mid H$ of $S$ to $H$. We observe that, since $S T$ is continuous, the map

$$
\left(x_{i 1}, \ldots, x_{i n}\right)_{i=1}^{K} \mapsto \sum_{i=1}^{K} S T\left(x_{i 1}, \ldots, x_{i n}\right):\left(E_{1} \times \ldots \times E_{n}\right)^{K} \rightarrow F
$$

is continuous, so its graph $G$ is a closed subset of $\left(E_{1} \times \ldots \times E_{n}\right)^{K} \rightarrow F$. Therefore $G$ is a complete separable metric space. Now the graph $\operatorname{Gr}(S \mid H)$ of $S \mid H$ is the image of $G$ under the continuous map

$$
\left(\left(x_{i 1}, \ldots, x_{i n}\right)_{i=1}^{K}, y\right) \mapsto\left(\sum_{i=1}^{K} T\left(x_{i 1}, \ldots, x_{i n}\right), y\right) \text {. }
$$

Therefore $\operatorname{Gr}(S \mid H)$ is analytic. By the Analytic Graph Theorem, Theorem 3.4, $S \mid H$ is continuous. Since $H$ is closed and of finite codimension, it follows that $S$ is continuous.

THEOREM 3.6. Let $A$ be a separable $F^{*}$-algebra with centre $Z$ such that $A Z$ is of corntable codimension in $A$. Then every positive functional on $A$ is continuous.

Proof. Let $f$ be a positive functional on $A$. Since $A Z$ is of countable codimension, so is $A^{2} Z$. We apply the previous lemma with $n=3, E_{1}=E_{2}=E=A, E_{3}=Z, F=\mathbb{C}, S=f$ and $T$ the multiplication map (so that Theorem 2.5 just says that $S T$ is continuous). The conclusion is that $A^{2} Z$ is closed and of finite codimension and that $f$ is continuous. (A similar argument shows that $A Z$ is closed and of finite codimension.)

COROLLARY 3.7. Let $A$ be a commutative, separable $F^{*}$-algebra. 
Then a necessary and sufficient condition for every positive functional on $A$ to be continuous is that $A^{2}$ be closed and of finite codimension (equivalently, that $A^{2}$ be of countable codimension).

Proof. By Theorems 3.6 and 1.3 .

\section{4. $F^{*}$-algebras with approximate identities}

In extending Varopoulos' theorem (Theorem 1.2 above) to $F^{*}$-algebras, we shall follow his approach of first proving a version of Cohen's Factorization Theorem and then combining it with the fact that the map $x \mapsto f(a x b)$ is continuous (our Theorem 2.1). The required version of Cohen's Theorem was first proved by Craw [5] for Fréchet (that is, complete, metrizable, LMC) algebras; another proof being given, using modules, by Summers [20]. We shall show here that Cohen's Theorem extends, in a particularly neat way, to complete, metrizable, locally convex (but not necessarily LMC) algebras. We follow Summers' approach.

Let $A$ be a locally convex $F$-algebra. Its topology may be defined by a countable set of seminorms $\left\{p_{i}: i=1,-2,3, \ldots\right\}$, and these may be chosen so that $p_{i}(x) \leq p_{j}(x)$ whenever $i \leq j$ and $x \in A$. Since the multiplication is jointly continuous, we may further adjust the choice of defining seminorms so that

$$
p_{i}(x y) \leq p_{i+1}(x) p_{i+1}(y) \quad(i=1,2,3, \ldots ; x, y \in A) .
$$

By a uniformly bounded left approximate identity in $A$, we shall mean a net $\left\{e_{\lambda}\right\}_{\lambda \in \Lambda}$ such that $e_{\lambda} a \rightarrow a$ for every $a \in A$ and there exists $K>0$ such that the set

$$
\left\{\left(K^{-1} e_{\lambda}\right)^{n}: \lambda \in \Lambda, n=1,2,3, \ldots\right\}
$$

is bounded in $A$. (Note that this agrees with Craw's definition in the LMC case.) By a left Fréchet A-module, we shall mean a left A-module $X$ which is an $F$-space (not necessarily locally convex), such that the multiplication map $(a, x) \mapsto \alpha x: A \times X \rightarrow X$ is separately (and hence jointly) continuous. We shall say that $X$ is essential if $A$ has a uniformly bounded left approximate identity $\left\{e_{\lambda}\right\}_{\lambda \in \Lambda}$ suç that $e_{\lambda} x+x$ 
for all $x \in X$. We shall let $d$ denote a translation-invariant metric defining the topology on $X$, and write $|x|=d(x, 0)(x \in X)$. (Note that $|\cdot|$ is subadditive, but that we do not have $|\alpha x|=|\alpha||x| \quad(\alpha \in \mathrm{C}$, $x \in X)$.)

THEOREM 4.1. Let $X$ be an essential Fréchet A-module. Then, for every $x \in X$ and every $\varepsilon>0$, there exist $a \in A, y \in X$ such that $x=a y, y \in \overline{A x}$ and $d(x, y)<\varepsilon$.

Proof. Let $\left\{e_{\lambda}\right\}_{\lambda \in \Lambda}$ be a uniformly bounded left approximate identity and $p_{i}(i=1,2,3, \ldots)$ seminorms, as described above. Let $A^{\sim}$ denote the algebra $A \oplus \mathbb{C} e$ obtained by adjoining an identity $e$ to $A$ in the usual way. To simplify notation, we shall write $a+\xi$ for $\left(a, \xi_{e}\right)$ $(a \in A, \xi \in \mathbb{C})$. The seminorms $p_{j}$ on $A$ extend naturally to $A^{\sim}$ by $p_{j}(a+\xi)=p_{j}(a)+|\xi| \quad(a \in A, \xi \in \mathbb{C})$.

Let $K$ be the uniform bound for $\left\{e_{\lambda}\right\}_{\lambda \in \Lambda}$, as above, and let $\rho>0$ be chosen so that $\rho /(1+\rho)<1 /(2 K)$. Then, for each $\lambda \in \Lambda,\left(1+\rho-p e_{\lambda}\right)$ is invertible in $A^{\sim}$. To show this, we must check that our hypotheses are sufficient for the usual argument to work. We have

$$
\left(1+\rho-\rho e_{\lambda}\right)(1+\rho)^{-1} \sum_{n=1}^{N}\left(\rho(1+\rho)^{-1} e_{\lambda}\right)^{n}=1-\left(\rho(1+\rho)^{-1} e_{\lambda}\right)^{N+1} \text {. }
$$

Since $\left\{\left(K^{-1} e_{\lambda}\right)^{n}: \lambda \in \Lambda, n=1,2,3, \ldots\right\}$ is bounded, we have, for each $j$, a constant $M_{j}$ such that

$$
p_{j}\left(\left(K^{-1} e_{\lambda}\right)^{n}\right)<M_{j} \quad(\lambda \in \Lambda, n=1,2,3, \ldots)
$$

Hence

$$
p_{j}\left(\left(\rho(1+\rho)^{-1} e_{\lambda}\right)^{N+1}\right)<2^{-N-1} \dot{M}_{j} \quad(j=1,2,3, \ldots),
$$

and so the right hand side of $(6)$ tends to 1 as $N+\infty$. Now the partial sums of $\sum_{n=1}^{\infty}\left(\rho(1+\rho)^{-1} e_{\lambda}\right)^{n}$ form a Cauchy sequence, since 


$$
p_{j}\left(\sum_{n=p}^{q}\left(\rho(1+\rho)^{-1} e_{\lambda}\right)^{n}\right) \leq \sum_{n=p}^{q} 2^{-n} M_{j} \quad(p \leq q, j=1,2,3, \ldots) .
$$

Hence the infinite sum converges and

$$
\left(1+\rho-\rho e_{\lambda}\right)^{-1}=(1+\rho)^{-1} \sum_{n=1}^{\infty}\left(\rho(1+\rho)^{-1} e_{\lambda}\right)^{n} \text {. }
$$

(Notice that in this well-known argument we have not required the seminorms to be submultiplicative, but we have used the local convexity of $A$.) We note that

$$
p_{j}\left(\left(1+\rho-\rho e_{\lambda}\right)^{-1}\right) \leq(1+\rho)^{-1} \sum_{n=1}^{\infty} 2^{-n} M_{j}=(1+\rho)^{-1} M_{j} .
$$

We shall choose a sequence $e_{1}, e_{2}, \ldots$ of elements of $\left\{e_{\lambda}: \lambda \in \Lambda\right\}$ inductively, beginning with $e_{1}$ which is chosen so that

$$
\left|\rho x-\rho e_{1} x\right|<\varepsilon / 2 \text {. }
$$

Suppose $e_{1}, \ldots, e_{n}$ have been chosen. We write

$$
\begin{aligned}
& t_{n}=\left(1+\rho-p e_{1}\right) \ldots\left(1+\rho-\rho e_{n}\right), \\
& h_{n}=t_{n}^{-1}, \\
& a_{n}=h_{n}-(1+\rho)^{-n} \in A, \\
& y_{n}=t_{n} x .
\end{aligned}
$$

Then we choose $e_{n+1}$ so that

$$
p_{n}\left(e_{n+1} a_{n}-a_{n}\right)<2^{-n}
$$

and

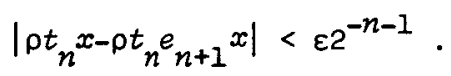

We can obtain (10) because $e_{\lambda} a_{n}+a_{n}$ and (11) because $\rho t_{n} e_{\lambda} \rightarrow \rho t_{n} x$, by continuity of scalar and module multiplication and the fact that $X$ is essential. Since the seminorms $p_{j}$ are increasing, (10) implies 


$$
p_{j}\left(e_{n+1} a_{n}-a_{n}\right)<2^{-n} \quad(1 \leq j \leq n) .
$$

We prove that $\left(a_{n}\right)_{n=1}^{\infty}$ is a Cauchy sequence. It follows from the definition of $\left(a_{n}\right)$ that

$$
a_{n+1}-a_{n}=\rho\left(1+\rho-\rho e_{n+1}\right)^{-1}\left\{(1+\rho)^{-n-1} e_{n+1}+e_{n+1} a_{n}-a_{n}\right\} \text {. }
$$

Hence, using (5),

$$
p_{j}\left(a_{n+1}-a_{n}\right) \leq \rho p_{j+1}\left(\left(1+\rho-\rho e_{n+1}\right)^{-1}\right)\left\{(1+\rho)^{-n-1} p_{j+1}\left(e_{n+1}\right)+p_{j+1}\left(e_{n+1} a_{n}-a_{n}\right)\right\} \text {, }
$$

for all $j$. Applying (8), (12) and the inequality $p_{j}\left(e_{\lambda}\right)<K M_{j} \quad(\lambda \in \Lambda$, $j=1,2,3, \ldots)$, which is a special case of $(7)$, we see that

$$
p_{j}\left(a_{n+1}-a_{n}\right) \leq \rho(1+\rho)^{-1} M_{j+1}\left\{(1+\rho)^{-n-1} K M_{j+1}+2^{-n}\right\}
$$

for $1 \leq j \leq n-1$. This shows that $\left(a_{n}\right)$ is a Cauchy sequence: we let $a=\lim a_{n}$. (Note that we have again used the local convexity of $A_{.}$)

We also have that $\left(y_{n}\right)$ is Cauchy, since

$$
\left|y_{n+1}-y_{n}\right|=\left|\rho t_{n} x-\rho t_{n} e_{n+1} x\right|<\varepsilon 2^{-n-1} \quad(n=1,2,3, \ldots) \text {, }
$$

by the definitions of $y_{n}$ and $t_{n}$ and inequality (11). We let $y=\lim y_{n}$.

The conclusions of the theorem are now clear. Since $h_{n} y_{n}=x$ and $y_{n}=t_{n} x$ for all $n$, we have $x=a y$ and $y \in \overline{A x}$. Finally,

$$
\begin{aligned}
d(x, y) & \leq\left|x-y_{1}\right|+\sum_{n=1}^{\infty}\left|y_{n+1}-y_{n}\right| \\
& \leq \varepsilon 2^{-1}+\sum_{n=1}^{\infty} \varepsilon 2^{-n-1}, \text { by (9) and (13), } \\
& =\varepsilon .
\end{aligned}
$$

COROLLARY 4.2. Let $A$ be a locally convex F-algebra with uniformly bounded left approximate identity. Then, for every sequence $x_{n} \rightarrow 0$ in 
$A$, there exists $a \in A$ and a sequence $y_{n} \rightarrow 0$ in $A$ such that $x_{n}=a y_{n}$ for alz $n$.

Proof. Let $c_{0}(A)$ denote the left Fréchet $A$-module of all zeroconvergent sequences in $A$ with the topology given by the seminorms

$$
P_{j}\left(\left(a_{n}\right)\right)=\sup _{n} p_{j}\left(a_{n}\right)
$$

corresponding to the topology-defining seminorms $p_{j}$ on $A$. Then (5) implies

$$
P_{j}(a x) \leq p_{j+1}(a) P_{j+1}(x) \quad\left(a \in A, x \in c_{0}(A)\right),
$$

and it is easy to check that $c_{0}(A)$ is essential. The corollary now follows by appling Theorem 4.1 with $X=c_{0}(A)$.

THEOREM 4.3. Let $A$ be a locally convex $F^{*}$-algebra with uniformly bounded left approximate identity. Then every positive functional on $A$ is continuous.

Proof. Let $x_{n} \rightarrow 0$ in $A$. Then, by Corollary 4.2, we may write $x_{n}=a y_{n}$ with $a \in A$ and $y_{n} \rightarrow 0$ in $A$. By a second application of Corollary 4.2, we can write $y_{n}^{*}=b^{*} z_{n}^{*}$ with $b^{*} \in A$ and $z_{n}^{*} \rightarrow 0$ in $A$. Thus, since * is continuous, we have $x_{n}=a z_{n} b$ with $z_{n} \rightarrow 0$. By Theorem 2.1, $f\left(x_{n}\right) \rightarrow 0$; hence the result.

\section{Problems}

In this final section, we list a few questions which we believe to be open.

5.1. Is it true that if $A$ is an $F^{*}$-algebra with $A^{2}$ closed and of finite codimension, then every positive functional on $A$ is continuous? Is this true under any of the following additional hypotheses on $A$ :

$$
\begin{aligned}
& \text { (i) } A \text { is Banach; } \\
& \text { (ii) } A \text { is separable; } \\
& \text { (iii) } A \text { is Banach and separable; }
\end{aligned}
$$


(iv) $A$ is commutative (but not separable)?

5.2. Does Theorem 4.1 (and hence Corollary 4.2 and Theorem 4.3) hold when $A$ is not locally convex?

5.3. A possible approach to 5.1 would be to try to obtain something like the conclusion of Corollary 4.2, that is, simultaneous factorization of a sequence, from a hypothesis such as $A^{2}=A$ which is weaker than the existence of a uniformly bounded left approximate identity. Specifically, we ask the following questions.

(i) Suppose that $A$ is a separable Banach algebra such that, for every $x \in A$, there exist $a, b \in A$ with $x=a b$ (and we may also require that $\|a\|\|b\| \leq K\|x\|$ for some fixed $K$ ). Is it then true that every sequence $x_{n} \rightarrow 0$ in $A$ may be written as $x_{n}=a y_{n}$ for some $a \in A$ and $y_{n} \rightarrow 0$ in $A$ ?

(ii) Suppose that $A$ is a separable Banach algebra with $A^{2}=A$. Then every $x \in A$ may be written in the form $x=\sum_{i=1}^{k} a_{i} b_{i}$ for some $a_{1}, \ldots, a_{k}, b_{1}, \ldots, b_{k} \in A$, where $k$ is a positive integer independent of $x$ (see [4], [12] or Lemma 3.5 (i) above). We also know that we may arrange that $\sum_{i=1}^{k}\left\|a_{i}\right\|\left\|b_{i}\right\| \leq K\|x\|$ for some number $K$ independent of $x$ [12]. Does it follows that every sequence $x_{n} \rightarrow 0$ in $A$ may be written in the form $x_{n}=\sum_{i=1}^{k} a_{i} y_{i n}$, for some $a_{i}, y_{i n} \in A \quad(1 \leq i \leq k$, $n=1,2,3, \ldots)$ with $y_{i n}+0$ as $n \rightarrow \infty \quad(1 \leq i \leq k)$ ?

(iii) Do (i) and (ii) hold even when the sequence $\left(x_{n}\right)$ is replaced by a pair $\left(x_{1}, x_{2}\right)$ ?

\section{References}

[1] Richard Arens, "Linear topological division algebras", Bull. Amer. Math. Soc. 53 (1947), 623-630. 
[2] N. Bourbaki, Éléments de mathématique. Livre III: Topologie générale. Chapitre IX: Utilisation des nombres réels en topologie générale (Actualités Sci. Ind. 1045. Hermann, Paris, 1948). See also: Nicolas Bourbaki, Elements of mathematics. General topology, part 2. Chapter 9: Use of real numbers in general topology (Hermann, Paris; Addison-Wesley, Reading, Massachusetts; 1966).

[3] J.P.R. Christensen, Topology and Borel structure (North-Holland Mathematics Studies, 10. Notas de Matematica, 51. NorthHolland, Amsterdam, London; American Elsevier, New York; 1974).

[4] Jens Peter Reus Christensen, "Codimension of some subspaces in a Fréchet algebra", Proc. Amer. Math. Soc. 57 (1976), 276-278.

[5] I.G. Craw, "Factorisation in Fréchet algebras", J. London Math. Soc. 44 (1969), 607-611.

[6] H.G. Dales, "Automatic continuity: a survey", BulZ. London Math. Soc. 10 (1978), 129-183.

[7] Peter Grant Dixon, "Generalized B*-algebras" ( $\mathrm{PhD}$ thesis, University of Cambridge, Cambridge, 1970).

[8] P.G. Dixon, "Scalar homomorphisms on algebras of infinitely long polynomials with an application to automatic continuity theory", J. London Math. Soc. (2) 19 (1979), 488-496.

[9] P.G. Dixon and D.H. Freml in, "A remark concerning multiplicative functionals on LMC algebras", J. London Math. Soc. (2) 5 (1972), 231-232.

[10] J. Hoffman-Jфrgensen, The theory of analytic spaces (Various

Publication Series, 10. Matematisk Institut, Aarhus Universitet, Aarhus, 1970).

[11] David L. Johnson, "Continuity" of positive functions on topological *-algebras" (Preprint, University of Arkansas, Fayetteville, 1980).

[12] Richard J. Loy, "Multilinear mappings and Banach algebras", $J$. London Math. Soc. (2) 14 (1976), 423-429. 
[13] Richard J. Loy, "Notes on analytic spaces" (Mimeographed notes, Australian National University, Canberra, 1976).

[14] Ernest A. Michael, Locally multiplicatively-convex topological algebras (Memoirs of the American Mathematical Society, 11. American Mathematical Society, Providence, Rhode Island, 1952).

[15] Isaac Namioka, Partially ordered linear topological spaces (Memoirs of the Americal Mathematical Society, 24. Americal Mathematical Society, Providence, Rhode Island, 1957).

[16] Michael Neumann, "Continuity of sublinear operators on F-spaces", Manuscripta Math. 26 (1978), 37-61.

[17] Shu-Bun $\mathrm{Ng}$ and Seth Warner, "Continuity of positive and multiplicative functionals", Duke Math. J. 39 (1972), 281-284.

[18] Ся До-шнин [Dao-Shing Shah], "О полунормированных нольцах с ннволюцией" [On semi-normed rings with involution], Izv. Akad. Nauk SSSR Ser. Mat. 23 (1959), 509-528.

[19] Allan M. Sinclair, Automatic continuity of linear operators (London Mathematical Society Lecture Note Series, 21. Cambridge University Press, Cambridge, New York, Melbourne, 1976).

[20] Marguerite K. Summers, "Factorization in Fréchet modules", J. London Math. Soc. (2) 5 (1972), 243-248.

[21] Nicholas Th. Varopoulos, "Sur les formes positives d'une algèbre de Banach", C.R. Acad. Sci. Paris 258 (1964), 2465-2467.

[22] W. Zelazko, "Metric generalizations of Banach algebras", Rozprowy Mat. 47 (1965), pp. 70.

Department of Pure Mathematics,

Hick Building,

The University,

Sheffield S3 7RH,

England. 